DOI: $10.30842 /$ ielcp230690152405

\author{
G. Blažienè \\ (Institute of the Lithuanian Language, Vilnius, Lithuania)
}

\title{
BALTISCHE SPUREN IN ORTSNAMEN EUROPAS?
}

\section{The Traces of the Balts in the European Onomasticon?}

It should be noted that the relations among many tribes, and later among nations, in ancient history are reflected not only in archaeological finds, but especially in the treasure trove of proper names. Significant progress has recently been made in finding answers to questions about the history of the population of Europe. On the other hand, the results of proper names studies sometimes do not coincide with, for instance, the historians' view when it comes to precisely defining tribal habitats and identifying traces of the Balts in Europe. The current research not only analyses proper names but also deliberately uses the term "ethnogenesis", despite the tendencies underestimating this concept.

It has long been shown that systematic studies of proper names, and especially hydronyms, may contribute to the elucidation of the most complex issues of ethnogenesis. However, in their interpretations of the nature of different cultures, researchers of proper names must closely follow the development of archaeological research. One must re-analyze the historical sources to obtain the results that would reflect the achievements of modern science. It is obvious that without proper names it is not possible to obtain accurate data on the history of the population of the area and the ethnic composition of its populace.

A particular attention needs to be paid to proper names identified in the contact areas of different nations, in this instance among the Baltic, Germanic, and Slavic tribes. Among the researchers of Pomeranian proper names, there are not as many substantial arguments in favour of the opinion that the proper names identified west of the Vistula are of Slavic origin, rejecting any fact proving their Indo-European and Baltic origins. An obvious example is the name of the river Persante, the origin of which may and must be clarified in the future in the light of new facts about the expansion of the Baltic or Prussian habitat. When investigating proper names, and especially hydronyms, it is important to consider the principles of their derivation and their morphological structure. The recent studies of Prussian proper names and archaeological findings are forcing us to reconsider, study and prove (archaeologists are already doing so in principle) that the area west of the Vistula should be considered the western boundary of the spread of the Baltic tribes. Recognizing the important role of the Germanic, Romance, and Slavic tribes in Europe and avoiding panBalticism, based on the important works of archaeologists and the rapidly deepening cognition analysis of ancient sources, the role of the Baltic tribes in Europe remains not yet fully comprehended by the linguists, but particularly relevant. 
Key words: the Balts, the Balts in Europe, the Vistula, western boundary of the Baltic tribes, proper names of residential areas (oikonyms), language contacts.

Г. Блажене

(Институт литовского языка, Вильнюс, Литва)

\section{Балтийский след в топонимике Европы?}

Отношения между многими племенами, а позднее и народами в древней истории отражаются не только в археологических находках, но и в географических названиях. В последнее время достигнуты большие успехи в изучении заселения Европы. С другой стороны, результаты анализа топонимов и гидронимов иногда не совпадают с доводами историков, особенно при попытках точно определить ареалы обитания племен и установления балтийского следа в Европе.

Уже давно показано, что системные исследования географических названий, в особенности гидронимов, могут быть весьма полезными для прояснения сложнейших вопросов этногенеза. При изучении этого материала необходимо следить за развитием археологической науки и проводить на современном уровне анализ исторических источников. Очевидно, что без изучения географических названий невозможно собрать точные данные, касающиеся этапов заселения Европы и этнического состава ее жителей в разные периоды.

Особенно пристального внимания требуют географические названия, засвидетельствованные в контактных ареалах. В статье исследуются отношения между балтийскими, германскими и славянскими племенами. Среди исследователей, изучающих историческую ономастику Померании, бытует не особо обоснованное фактами мнение о славянском происхождении топонимов, зафиксированных к западу от Вислы. При этом их возможное индоевропейское и балтийское происхождение часто не рассматривается. Яркий пример - название реки Persante, происхождение которого с учетом новых фактов о расположении ареала обитания балтов или пруссов в будущем может и должно быть уточнено. При изучении географических названий, в особенности гидронимов, важно учитывать особенности словообразования и их морфологическую структуру. Новые исследования прусских географических названий и археологические находки заставляют заново осмыслить, изучить и доказать (отчасти это делают археологи), что территорию к западу от Вислы следует считать западной границей ареала распространения балтийских племен. Признавая важную роль германских, романских и славянских племен в Европе, избегая панбалтизма и опираясь на значимые работы археологов и стремительно углубляющийся анализ античных источников, нам все же приходится констатировать, что вопрос о роли балтийских племен в Европе еще не до конца осмыслен языковедами.

Ключевые слова: балты, ареал проживания балтов в Европе, Висла, западная граница балтийских племен, гидронимы, названия населенных пунктов (ойконимы), языковые контакты. 
Am Anfang ist kurz zu betonen, dass die ersten Beziehungen der Frühgeschichte vieler Stämme, später der Völker, sich nicht nur im archäologischen Befund, sondern auch besonders im Namenschatz niedergeschlagen haben. Unser Erkenntnisstand der Besiedlungsgeschichte von Europa ist einerseits erheblich vorangeschritten, andererseits geraten mit dieser Geschichte verbundene Ortsnamen in einen Widerspruch zu ihren Forschungsergebnissen, wenn man möglichst genau die Frage zum Stammesgebiet beantworten und die baltischen Spuren festlegen möchte.

Die vorliegende Untersuchung berücksichtigt nicht nur die Eigennamen, sondern greift auch kurz den Termin der Ethnogenese auf. Dieser Termin wird bewußt verwendet, obwohl die Tendenzen der Verwertung des Begriffes und mit ihm entstehenden Fragen gut bekannt sind. Die Konzeption der Ethnogenese wurde in vielen Fällen zur verwickelten Angelegenheit, um sie ohne Weiteres zu lösen. Manchmal wurde sie auch durch politische Aspekte gefärbt. Herwig Wolfram (2008: 790; s. 2011: 362f.) betont, dass „Heute wie einst bedeutet Genese/Genesis sowie Ursprung, origo, Werden und Entstehung, während mit Ethnos in den meisten Sprachen eine pränationale Formierung unbestimmen Entwicklungsgrades eine Minderheit oder Unterabteilung moderner Nation, aber sicher keine genetische Gruppe gemeint ist. Wie Ethnogenese ist heute selbstverständlich auch die ethnische Indentität umstritten. Eine Ethnogenese ist dannn erfolgreich, wenn es einen gentilen Sondernamen hervorgebracht hat, wenn es nicht heißt ,die Slawen, sondern die Böhmen (Mährer, Karantanen) genannt werden“ (s. dazu Graus 1980: 179f., 157). Der berühmte deutsche Historiker Reinhard Wenskus (1961 [1977]: 246-255) hat in seinem Buch, dessen Begriffe bis heute heftig diskutiert werden und sich mit ihnen viele berühmte Autoren der Gegenwart in Sammelbänden auseinandersetzen, den Begriff der Ethnogenese nur einmal verwendet bei der Benennung eines kleinen Kapitels Die Kulturverbände und die Ethnogenese der Germanen (246-255). Hervorzuheben wäre 2002 erschienene Sammelband Gillet Andrew (ed). On Barbarian Identity: Critical Approaches to Ethnicity in the Early Middle Ages. Studies in the early Middle Ages, Vol. 4. (s. in diesem Buch Gillet 1-18, 85-121; Goffart 21-37; Murray 39-68; Pohl 220-239), in dem man im Grunde genommen $\mathrm{zu}$ den von Wenskus hervorgehobenen Ideen Stellung genommen wird. Michael Kulikowski (2002: 69) betont mit einer gewissen Dose von Humor, dass die Theorie von der Ethnogenese im Unterschied zu den Barbaren die Atlantik überquert hatte und fügt hinzu: Diese Analogie ist nur 
teilweise komisch: es ist eine seltene und überraschende Sache, wobei das akademische Konstrukt seine Erfolgsmetapher zur Verfügung stellt. Das war ein Erfolg: zurzeit wird in den USA über die Ethnogenese in den Lehrbüchern für Westzivilisation und in den Trendzeitschriften für Stipendienbewerbungen geschrieben. 2011 verallgemeinerten die Mitarbeiter des Lehrstuhles für Mediävistik Daniel Ziemann und Balázs Trencsényi der Zentraleuropäischen Universität (Central European University) in Budapest das Programm für Studierende. Die Autoren haben unterstrichen, dass das Poblem der Ethnogenese die Hauptfrage ist, um die Modelle der modernen Historiographie des frühen Mittelalters zu verstehen. Der Zusammenhang der politischen Umstände und der Forschungsrichtungen hilft bei der Klärung der Frage, ob die historischen Debatten bei der Behandlung der Tatsachen der Vergangenheit einen politischen Charakter tragen s. http://archive.ceu.edu/node/ 24561 (Zutritt: 0504 2020). Sehr treffend erforscht Jurij K. Kusmenko (2011) die komplizierten Fragen des frügermanischen Ethnos gestützt auf Angaben der Linguistik, Archäologie und Genetik. Wolfram (2010: 14) betont ausdrücklich, dass die beliebte Frage nach der Herkunft und Urheimat der Völker mit Hilfe herkömmlicher historischer Fragestellung nicht zu beantworten ist, weil für die antiken Ethnographen ein Volk erst dann existierte, wenn es in den Geschichtskreis der zivilisierten Welt getreten war. Eben in diesem Zusammenhang könnte man einige Gedanken in Erwägung ziehen, wann sind die baltischen Völker von der zivilisierten Welt erwähnt worden, angefangen von Tacitus in „Germania“ um das Jahr 98 als Aestiorum gentes, Ästier, oder in „Geographia“ von Claudius Ptolemäus in der Mitte des 2. Jhs. erwähnten zwei baltische Stämme Galindae und Sudeni, also Galinder und Sudauer, die frühesten bezeugten Ethnonyme der Ästier von Tacitus (s. dazu die Forschungen von Eugenijus Jovaiša 2012: 58ff.; 2014: 121ff.; 2019: 19ff., in denen auch der neueste ausführliche Forschungsstand angeführt wird.). Gestützt auf Florin Curta (2001: 6-35) ${ }^{1}$ betont

\footnotetext{
${ }^{1}$ Es wurde beschlossen auch diese Überlegungen anzuführen, damit die Leser selber Schlussfolgerungen ziehen könnten. Man ist nicht im Klaren, ob für die Wissenschaft entscheidend ist, die Interpretationen vorzulegen, ohne Vorurteile auf die Tatsachen einzugehen. Die Geschichte der Slawen wird heute meistens von den Historikern und Archäologen geschrieben, vor fünfzig Jahren wurde aber der autoritative Diskurs von den Fachleuten der vergleichenden Sprachwissenschaft bestimmt. Die Zusammenwirkung der unter verschiedenen Disziplinen entstandenen Betrachtungen hat den Begriff der (slawischen) ethnischen Zugehörigkeit zu einem mächtigen
} 
Wolfram (2008: 787), dass offenbar die russische Sozialforschung den Begriff „Ethnogenese“ in die Wissenschaft einführte.

Laut Walter Pohl (1998: 20) haben die Wissenschaftler der Gegenwart, genauso wie die Ethnographen der Antike, versucht, die Ethnizität objektiv zu bezeichnen mit Hilfe der Sprache, Kultur und der Bräuche, sowie des Territoriums oder der politischen Verfassung, obwohl ihre relative Bedeutsamkeit diskutiert wurde. Pohl folgt in seinen Überlegungen dem Gedankengang einiger Vorgänger, darunter auch dem von Wsewolod W. Isajiw (1993: 415) ${ }^{2}$.

Längst ist bewiesen, dass systemhafte Untersuchungen der Eigennamen, insbesondere der Gewässernamen, wesentlich zur Klärung der kompliziertesten Fragen der Ethnogenese beitragen können. Die Autorin des Beitrages versteht die Ethogenese als Prozess, als Werden. Bei der Begründung der Natur unterschiedlicher Kulturen dürfen die Namenforscher keinesfalls die Entwicklung des archäologischen Gedankens außer Acht lassen. Auch die wiederholte Analyse der historischen Quellen nach dem heutigen

Werkzeug der „Kulturpolitik“ umgewandelt. Das Vorhandensein des Verhältnisses zwischen Nationalismus einerseits und der Historiographie sowie Archäologie andererseits, ist keine neue Idee. Es bleibt aber unklar, welche Bedeutung dieses für die (slawische) ethnische Zugehörigkeit (obwohl das Wort von den sich mit der „Kulturpolitik“ befassenden Wissenschaftlern entweder sehr selten oder überhaupt nicht gebraucht wurde) gehabt hat. Der Einblick in die neueste Literatur über die ethnische Zugehörigkeit und die Rolle der materiellen Kultur zeigt, inwiefern der historiographische Diskurs der Frühslawen mit den gegenwärtigen anthropologischen und in manchen Fällen auch archäologischen Untersuchungen übereinstimmt. Die Leser sollen entscheiden, ob diese Behauptung stimmt, dass die sovjetischen Anthropologen der Meinung waren, dass die ethnischen Gruppen (Ethnose) im Laufe der Formierung der Ethnogenese und der Geschichte unverändert geblieben sind, und schlugen vor, dass die ethnische Einheit im sozialen und politischen Vakuum entstehen werden sollte.

${ }^{2}$ Ungeachtet mancher gegenwärtiger Überzeugungen, muss den Ausgangspunkt des Herkunftskonzeptes der Ethnizität die Idee einer unterschiedlichen Kultur ausmachen. Die Kultur wird hier teilweise im traditionellen Hinblick auf den anthropologischen Sinn verstanden und zwar, als eine, die ganze Lebensweise umfassende Erscheinung. Allerdings weist die gemeinsame Lebensweise nicht unbedingt nur die Gesamtheit alltäglicher Bräuche hin, obwohl sie einbegriffen werden können. Sie umfasst eher eine sonderartige historische Erfahrung einer Gruppe. Die Kultur ist daher grundsätzlich ein Verschlüsselungssystem von symbolhaften Modellen solcher Erfahrung. Es ist belanglos, wie sich die Elemente eine Kultur von denen einer anderen unterscheiden. Eine einzigartige Kultur besteht in einer einzigartigen historischen Erfahrung bestimmter Gruppe. Ihr Erzeugnis ist das Gefühl (der Sinn) der eigenartigen Menschlichkeit. 
Stand unseres Wissens ist unbedingt erforderlich. Schon 1930 stellte Max Vasmer (507) fest, daß die slawische Urheimatfrage in erster Linie durch gründliche Lehnwörter- und Orstnamenforschung gefördert werden kann. 2014 unterstrich Jürgen Udolph (11311144), ohne auf den Begriff der Ethnogenese zu verzichten, erneut und sehr überzeugend die Rolle der Namen im Dienste der Geschichte sowie in jeder sprachlichen Erforschung einer Region. Des Weiteren kam von allen Namenforschern anzunehmende Bemerkung zustande, dass man in erster Linie von den Fluss- und Ortsnamen auszugehen ist.

Es steht fest, man kann ohne Eigennamen keine Erkenntnisse für die Besiedlungsgeschichte und ethnische Zusammensetzung der Bevölkerung gewinnen (Wenzel 2015: 38). Man hat z.B. in Niedersachsen auch Ortsnamen, die man nur mit Hilfe des Baltischen erklären kann. Aber dann sind es keine baltischen Namen, sondern die Appellativa und sprachlichen Relikte, die nur im Baltischen ihre Entsprechung finden. Man muss dann davon ausgehen, dass von indogermanischen oder frühgermanischen Sprechern Wörter genutzt wurden, die in ihrer Sprache noch vorhanden waren. Später starben diese Wörter - die Namen lebten weiter - und Entsprechungen zu diesen Wörtern kann nur noch im Baltischen finden (vgl. Casemir, Udolph 2006: 114-144).

Leider ist es kaum möglich im Rahmen des Beitrages die baltischen Eigennamen in ganz Europa sogar in groben Umrissen vorzustellen. Deshalb wurde beschlossen, sich auf das Gebiet östlich und westlich der Weichsel zu beschränken, um wenigstens noch einmal einen Versuch einzugehen zur Bestimmung der möglichen Auszugsgebiete der Balten mit Hilfe der Namen.

Die Weichselmündung und der Unterlauf der Weichsel werden traditionell für westliche Grenze der Balten gehalten, die intensiv im 4. Jahrzehnt des vergangenen Jahrhunderts die Aufmerksamkeit der Sprachforscher und Archäologen auf sich gezogen hat mit dem Vorschlag diese Grenze westlich der Weichsel zurückzulegen. 2012 vermochte der Archäologe Jovaiša neues Licht auf den Verband der Ästier mit den Goten zu werfen auf beiden Seiten der Weichselmündung, später Pommern, noch später auf einen großen Teil des rechten Weichselufers. Hier siedelten deutsche, polnische und andere Archäologen die ostgermanischen Goten an, ungeachtet des von Sprachforschern festgestellten Faktums, dass sich ein Großteil Pommerns, die Weichsel samt ihrem Unterlauf und auch noch Gebiet zwischen der Weichsel und dem Pregel auf dem Verbreitungsgebiet baltischer Gewässer- und anderer Ortsnamen 
befinden. Die baltische Welt ist früher sichtbar als die germanische, geschweige von der slawischen.

Allein für sich genommen können die Namen nicht alles aussagen ohne geschichtliche Kenntnisse über die Bewohner bestimmter Gebiete und über den Lebensrhythmus der Kulturen von Nachbarn (vgl. Marija Gimbutienè (1985: 21ff.), die die Ansicht vertritt, dass baltische Gewässernamen zwischen Moskau und Berlin $\mathrm{zu}$ finden sind. Aber aus der Form des Namens ist oft nicht ersichtlich, welchem Ethnikum die Namengeber angehörten.).

Besonders präzise zu erforschen sind die Eigennamen in den Berührungsgebieten unterschiedlicher Völker, in unserem Fall baltisch-germanisch-slawisch. Unter den vielen Forschern zu den Namen in Pommern, westlich der Weichsel, herrscht schon seit geraumer Zeit das Streben vor, die Namen als slawisch zu betrachten und die alteuropäische (indogermanische) und baltische Schicht auszuschließen. Das anschaulichste Beispiel ist der Flussname Persante, dem man im Lichte der Erweiterung des baltischen bzw. altpreußischen Materials neue Deutungen zuschreiben könnte, damit die Namenkunde gesicherte Aussagen machen könnte. Dass Germanen, Romanen und Slawen Anteil an Europa haben, steht außer Zweifel. Im Lichte der neuesten Erkenntnisse in der Archäologie und Namenforschung wird die Beantwortung der Frage, welche Rolle die Balten in Europa gespielt haben, ohne dem Panbaltismus zu verfallen, auch nach den Stammsitzen der Balten für viele Forscher immer klarer, für andere kaum sogar nach dem heutigen Erkenntnisstand.

Die Ergebnisse der neuesten Forschungen zu den apr. Eigennamen sowie den baltischen Stämmen zuzuordnende archäologische Funde werfen erneut die Frage auf, ob die Weichsel als Westgrenze der Balten anzusehen ist (s. sehr ausführliche und gut belegte Analyse der antiken Qellen über die Weichsel von Darius Alekna 2019: 251-271).

Die Festlegung der Weichsel als der Grenze der Balten und die Bestimmung des Umfanges der baltischen Besiedlung um diesen Fluss hat die Gemüter der Historiker, Archäologen, Geographen und nicht zuletzt der Namenforscher seit der 1. Hälfte des 20. Jhs. bewegt. Ein treffender Überblick wird von Darius Ivoška (2016: 208ff.) geboten, aber die noch früheren Erkenntnisse zu diesem Thema wurden außer Acht gelassen. Die erlauben mit dem Überblick schon seit dem Ende den 19. Jhs. anzufangen. Oskar Beyersdorf (1881: 25-64, hier S. 51f.) erläutert unter anderen Gewässernamen auch den Flussnamen die Persante, Parsand, Parsant, 
Parzandi, Parzan, Persandi, Persanta und stellt ihn zu poln. prośnica usw. Es wird versucht, eine Art von Semantik und Motivation des Namens im Sinne des Autors vorzuschlagen, indem er betont, dass das Wasser des Flusses rein und ungemischt war und man könnte den Wasserlauf als prèsina woda, d.h. 'Frischwasser' bezeichnen. Des Weiteren werden die schon angegeben Tatsachen nicht wiederholt, sondern nur die hervorgehoben, auf die man von einem anderen Blickwinkel eingehen kann.

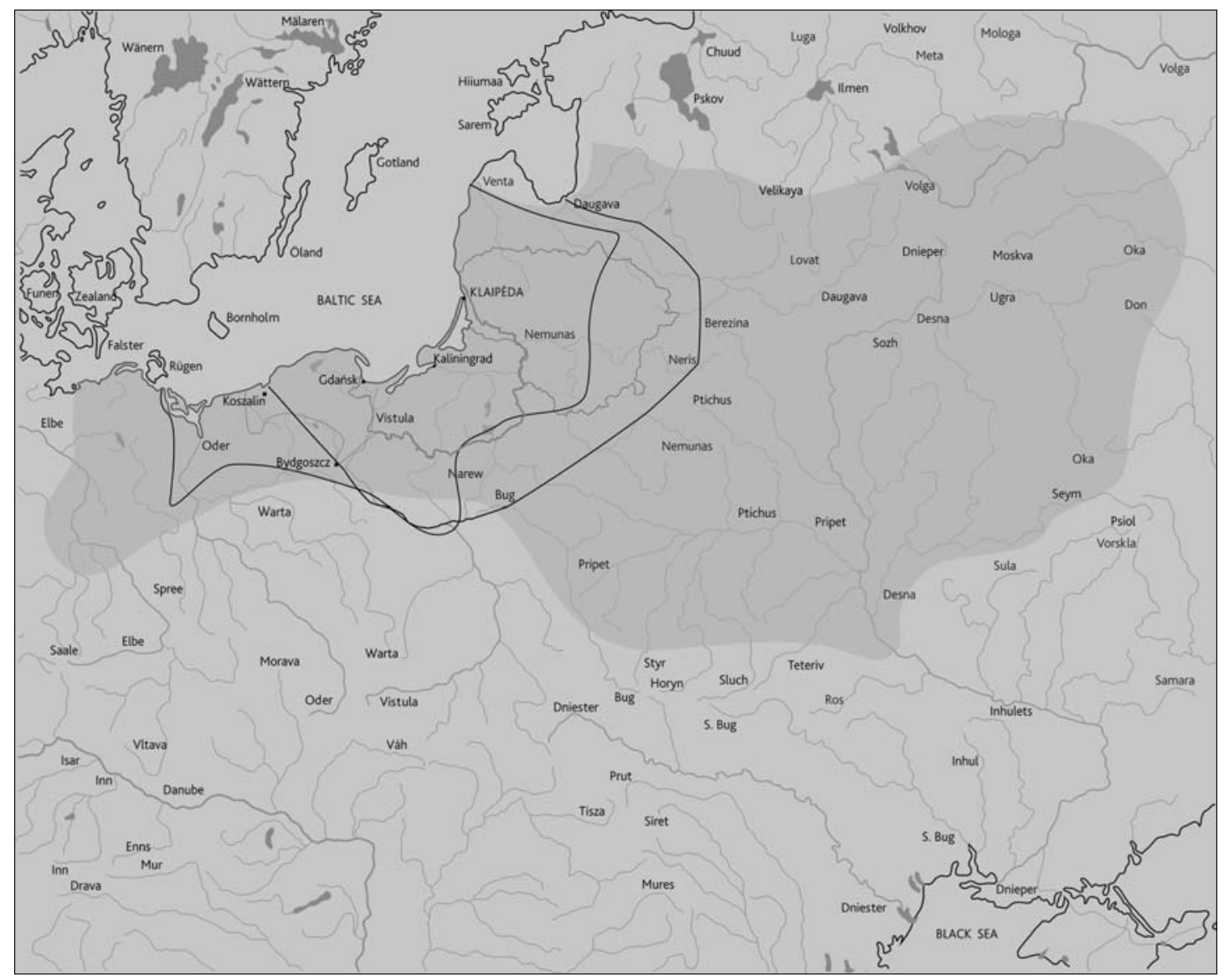

Karte Nr. 1. Die Grenzen der westlichen Balten nach Karl Engel und Lothar Kilian. Autor E. Jovaiša.

1932 stellt Robert Holsten (1-5, hier von der S. 3) bei der Behandlung der illyrischen Fluß- und Ortsnamen in Pommern fest, dass der Flussname Persante in Hinterpommern eben $\mathrm{zu}$ den illyrischen Namen gehört und geht in erster Linie von der Derivation aus, d.h. von dem Suffix $n t$ - in der Form von -ant-. Dieses Suffix haben viele illyrische Ortsnamen sowie die lit. Gewässernamen Laukante, Salantas (ibd. 4-5). Übrigens führt der Autor auch die historischen Belege von Persante auf, gestützt auf die Bände des „Preußischen Urkundenbuches“. 1933 veröffentlicht der Sprach- 
forscher Friedrich Lorentz (49-59) eine Liste der Ortsnamen des Untersuchungsgebietes, die nach seiner Überzeugung altpreußisch sein könnten: 1. Danzig, poln. Gdańsk, kasch. Gdańsk, urk. 997 Gyddanyzc zu pr. gude (err. gudde 'Gebüsch, Wald'), s. NMP III 110f., vgl. DONB 119, wo die slawische Herkunft als möglich vorgestellt wird, von urslaw. gъde- 'nass, bewachsen' und die apr. Herkunft nicht ausgeschlossen wird; 2. Gdingen, poln. Gdynia; 3. Mottlau, poln. Mottawa, urk. 1280 Motlaua, 1283, 1292, 1295 Mutlaua, 1307, 1317 Mutlawa, 1308, 1310 Mutholowo, 1338 Mutelow, Mutlow usw., vgl. lit. mutulas 'die Blasen beim kochenden Wasser' (derselben Meinung war Hubert Górnowicz noch 1985: 20f.); 4. Ohra bei Danzig, poln. Orunia, Orania, urk. 1338 Oran, 1338 Ore, 1356, 1438 Orana, vgl. den lett. Flussnamen Aruona, apr. *Arôna (ausgehend von lautlichen Besonderheiten ordnen Górnowicz (1985: 22f.) und Udolph (1990: 219ff.) mit Sicherheit diesen FIN der alteuropäischer Schicht zu. Bei Zbigniew Babik (2001: 409) stießt man auf eine ganz andere Erklärung, die man unsicher halten könnte; 5. Jetau, Kr. Danziger Höhe, poln. Jagatowo, urk. 1352 Jodotowitz, 1374 Jodotowicz, 1400 Judotowicz zu einem apr. PN Jodothe (eindeutig wird dieselbe Meinung von den Autoren von NMP IV: 27 angenommen.); 6. Groß- und Klein-Saalau, Kr. Danziger Höhe, poln. Żuława, kasch. Zēlava, urk. 1398 Solow, Solaw, 1421 Zolow, hinzufügen wäre 1437/38 Solaw das Dorf hot 30 1/2 huben 6 morgen und 1 firtel... (OF 131 324), vgl. apr. soalis 'Kraut'. Die Belege bestätigen die apr. Herkunft; 7. Nassenhuben, Kr. Danziger Niederung, urk. 1384, um 1400 Mudestentz, 1524 Mutterstrincze später Mutterstrentz. Der ON wird als slaw. betrachtet und vorsichtig zu apr. moter 'Beritt' gestellt (zu moter s. Gerullis 1922: 98; Blažienè 2000: 98) (S. 53f.) Laut NMP VII: 224f. heißt der Ort Mokry Dwór und bleibt ohne sichere Etymologie. Er werden keine überzeugende Argumente für deutsche Herkunft dargeboten, um die altpreußische abzulehnen; 8. Schönau (Kr. Danziger Niederung, poln. Szonowo, 1308 Sunowo, 1310 Sonowo, vgl. apr. sunis 'Hund' (Górnowicz (1980: 156) nimmt den Vorschlag von Lorentz an und geht von einem apr. PN *Sunis oder apr. Appellativum sunis aus.); 9. Trutenau (Kr. Danziger Niederung, poln. Trutnowy, urk. um 1400 Trutenaw, Trutenow, 1421 Trawtynaw zu dem apr. PN Truthna (s. Gerullis 1922: 187; vgl. Przybytek 1993: 309; Blažienè 2000: 165); 10. Utatilo im Danziger Werder, urk. 1308 Vthatino, 1310 Uthatino zu einem apr. PN *Utato, *Utote, vgl. den apr. PN Vtil (Trautmann 1925: 111) (S. 55); 11. Uruti im Danziger Werder urk. 1308 Vruti, 1310 Vruthi, 1310 
Vruthy wohl zu einem apr. PN, vgl. (?) den apr. PN Orute (S. 56). Nicht zuletzt beweisen die von der Autorin hinzugefügten Kommentare, dass zwischen mehreren von Lorentz vorgestellten Namen am ehesten ein unmittelbarer Zusammenhang mit dem Altpreußischen besteht (s. Toporov 1983: 264).

1966 wurde in ziemlich umfangreichen Beitrag von Lorentz (243-250) posthum noch einmal zu apr. ON im Raum links der unteren Weichsel Stellung genommen. Nach Lorentz zogen die Ästier vom 5. Jh. in das Weichselland ein und bewohnten die Gegend zwischen Gdingen-Danzig-Schöneck-Stargard-Subkau. Die Forschungen der Gegenwart bieten andere Ergebnisse und in der Materialbearbeitung im Wesentlichen fortgeschritten ist. Die Zeitangaben siehen anders aus. Auf der Grundlage von Forschungsleistungen in der Onomastik kann man viel mehr beweisen. Mit Recht stützt sich Lorentz auf die Hilfe der ON und stellt fest, ,dass besonder häufig die Flußnamen einer früheren Bevölkerung angehören“" (ebd. 245). Der Autor bietet zwei FIN, die apreußischer Herkunft sind. Das sind die Namen der Flüsse - Mottlau (poln. Mutlaw), den er zu lit. mùtulas 'Wasserblase' und lett. mutulis 'Sprudel' stellt. Dieser Deutungsversuch wurde durch die apr. Seenamen Mutilis und Muteling bei Georg Gerullis (1922: 103), den lett. FIN Mutulīte (Schmid 1987: 7; Schmid 1989: 123) unterstützt (s. Górnowicz 1985: 20f.; vgl. Babik (2001: 177f.), der in diesem Fall keine gewaltigen Widersprüche erhebt.) und Orana, jetzt poln. Oruński Potok, und zieht wie im Jahre 1939 zur Hilfe den lett. FIN Aruona heran und kommt noch einmal zur Schlussfolgerung, dass es apr. *Arona gegeben hat. Zu diesem FIN s. oben.

Die Archäologen haben die Bedeutung der Eigennamen immer erkannt und sie als Zeugen der nicht genügend belegten Geschichte für ihr Forschungsfeld angesehen. Der Archäologe Lothar Kilian (1939: 67-68) hat fragend einige ON links der Weichsel aufgelistet: die FIN Persante und Radaune und die Siedlungsnamen Saulin, Labehn und Labuhn, Powalken, Straduhn, Rutzau, Karwen. Hans Krahe (1943: 11-12), der Autor des Begriffes Alteuropa, der entweder annerkant oder heftig diskutiert wird, stellt die Verbreitung der baltischen Ortsnamen westlich der Weichsel fest und behandelt im Grunde genommen 2 Namen, den FIN Persante und den Siedlungsnamen Paculent, bei deren Deutung Holsten für das Illyrische plädierte. Krahe formuliert folgende Hypothese: bei der Deutung von Paculent wäre an lit. pakulos 'Hede, Werg' zu denken und bei Persante an idg. *pers- 'sprühen, stieben, spritzen', vgl. idg. *preus- 'sprühen, spritzen' (LIV 2001: 493f.). 
1966 verallgemeinert Vladimir N. Toporov (104f.) bisherige Erkenntnisse $\mathrm{zu}$ diesem Thema und erwähnt am Ende seiner Ausführungen, dass die Deutung der ON im Untersuchungsgebiet nur anhand der baltischen Sprachen möglich ist. Was viele ON zwischen der Weichsel und der Elbe anbelangt, bleibt festzuhalten, dass ihre Herkunft nur durch das baltische und slawische Sprachmaterial bestätigt sein kann.

Unter der Anzahl weiterer Arbeiten sind die von Górnowicz (1980, 1985) zu erwähnen. Ein gewisses Gewirr haben die Büchlein des Pfarrers von Karthaus (poln. Kartuzy) Wilhelm Brauer, erschienen 1983 und 1988, in der Namenkunde hervorgerufen. Sie haben entweder die Aufmerksamkeit verdient, wie im Falle von Toporov (1983: 268-273; s. noch 1984: 8; wo im Literaturverzeichnis auf zwei Büchlein von Brauer hingewiesen wird.). An dieser Stelle ist angebracht zu sagen, dass es ist nicht gelungen ist, die erwähnten Arbeiten von Brauer zu finden. Höchstwahrscheinlich hat Brauer noch nicht druckfertige Werke dem berühmten Gelehrten geschickt. Toporov (1983: 273) drückt seine Dankbarkeit für das Zukommen beider Werke aus und sagt eindeutig, dass eine Ausgabe handschriftlich sei. Ob sie erschienen ist, konnte man keine Bestätigung finden. Am ehesten hat Toporov auch im Literaturverzeichnis des 4. Bandes von Prusskij jazyk die Handschriften oder die Druckvorlagen, wenigstens von 1983 erschienenen Büchleins von Brauer, angeführt. Im Literaturverzeichnis des 5. Bandes (1990: 9) tritt schon das Büchlein von Brauer, herausgegeben 1983, dem Leser entgegen. Toporov hat allem Anschein nach nie an der Zuverlässigkeit des Materials von Brauer gezweifelt.

Man darf die Tasache nicht vergessen, dass das Geleitwort zu der Brauerischen Ausgabe von 1988 hat Wolfgang P. Schmid (7-9) geschrieben und unterstrichen, dass die Streifzüge des Pastors durch seinen Heimatkreis Karthaus in einer internationalen wissenschaftlichen Diskussion aufzugreifen wären. Es sollte an dieser Stelle gesagt werden, dass die Hoffnung bestand, dass die polnischen Namenforscher in ihrem Ortsnamenwörterbuch Nazwy miejscowe Polski mit Brauer diskutieren werden. Die Diskusion fand nicht statt. Der Familienname Brauer fehlt im Literaturverzeichnis des Wörterbuches, obwohl er die Ergebnisse der Forschungsentwicklung präsentieren könnte. Aber die Büchlein von Brauer haben das Interesse nicht nur von Toporov oder Schmid erregt, sondern wurden auch ziemlich ausführlich in den Rezensionen von Udolph (1988) und Friedhelm Hinze (1989), dem bekannten Slawisten, Kaschubologen und Baltisten, besprochen. Der erstere umschreibt 
das Büchlein als sehr anregend für den Fachmann (hier S. 300), der zweite läßt keinen Stein auf dem anderen, indem er betont, dass der Autor mehr finden wollte, als die sprachliche Hinterlassenschaft der Altpreußen in dieser Region das zuläßt (hier S. 129). Die Autorin des Beitrages vermisst bei Brauer zuverlässiges Belegmaterial und vertrauenswürdiges Modell onomastischer Sprachkontaktforschung.

Das Thema „Baltische ON links der Weichsel“" hat die Lettin Valija Dambe angeschnitten. Dambe hat nach den Baltismen im Atlas von Heinrich Kiepert Atlas antiquus, zwölf Karten zur alten Geschichte gesucht und Schlussfolgerung gezogen, dass einige hypothetische Baltismen sogar westlich der Elbe auffindig sind: Ethonyme Sigingae und Boji, Ortsname Bojohaemum, Bergnamen Sudeti und Semana und Flussname Sala. Um das zu annehmbar zu beweisen, sollte man viel mehr über Verlauf, Dauer und Systemhaftigkeit des Integrationssprozesses von Namen erforschen und sich auf sehr zuverlässige Buchungen der Namen in historischen Quellen stützen (s. dazu Karlheinz Hengst 1985: 809-822). Dambe möchte manche von der erwähnten Onyme sogar für wahrscheinlich baltisch halten, obwohl Schmid (1981: 5) vor der Gefahr gewarnt hat, in einen Panbaltismus abzurutschen, wenn man baltische ON an der Elbe sucht. Und das mit Recht, es kann bei der Forschung des Namenschatzes, wobei das sichere und zuverlässige empirische Material notwendig ist, und in der Wissenschaft überhaupt nichts Schlimmeres passieren, als die Umwandlung der unklaren Tatsachen in die klaren. Die Möglichkeit und Notwendigkeit jeglicher grundlegend argumentierten Hypothesenerhebung sind nicht auszuschließen.

Krystyna Szcześniak hat (1993: 166-171) unbekannte baltische Bezeichnungen von Orten aus Masuren, Ermland und dem Oppelner Schlesien im Thorner Nachlass von Martin von Gerss untersucht. Die Namenforscherin hat festgestellt, dass im erwähnten Nachlass aus dem Goldaper Kreis 168 Siedlungsbezeichnungen aus den Jahren 1861 bis $1865 \mathrm{zu}$ finden sind, die bisher niemals notiert wurden. Uns würden besonders die Namen vom Oppelner Schlesien interessieren. Pietro Umberto Dini (2019: 54-56) äußert sich auch zu der westlichen Grenze der Balten aus und stellt fest, dass die Frage nach der westlichen Grenze der Balten noch immer aktuell ist.

Bis jetzt erfolgte keine passende Reaktion auf interessante Überlegungen von Václav Blažek (2003: 14-20) zu den möglichen baltischen Spuren in der Onymie von Ostböhmen. Der berühmte tschechische Indogermanist und Namenforscher führt baltische Versionen von 12 Gewässernamen und 4 Siedlungsnamen an. Die 
FIN Ledhuje ir Metuje, gestützt einerseits auf Topoelemente bzw. Topomorpheme, andererseits auf Derivation und morphologische Struktur, betrachtet der Sprachwissenschaftler nicht als alteuropäische GN, sondern als baltische. Das könnten Spuren von Galindern in Ostböhmen sein, d.h. Spuren ihrer Migration aus dem Territorium in Nordpolen nach Nordwesten Europas. Angeführte neue etymologische Interpretationen, die auf das baltische Material stützen, können zu der neuen Auffasung der Grenzen vom baltischen Areal führen. Sie können mehr oder weniger hypothetischen Charakter tragen, aber sie zu übersehen wäre kaum möglich. Diese Grenzen können anders angelegt werden, sie können sich verändern und die von Toporov formulierte Hypothese zur südwestlichen Richtung der Galinderbewegung unterstützen.

An dieser Stelle ist angebracht zu betonen, dass die GN und ON mit zuverlässigen Buchungen, unterstützt mit den Angaben von Archäologen und Erwägungen von Historikern behilflich sein können, genauer die westliche und die südwestliche Grenze der Balten festzustellen.

Die Karte oder das Schema von Kilian (1960: 47) spiegelt die Verbreitung der baltischen Namen links der Weichsel und somit die Ausweitung des Siedlungsareals der Balten wider.

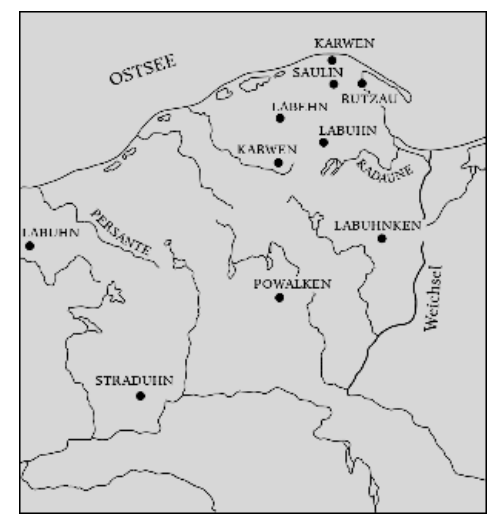

Zahlenmäßig ist das Schema nicht reich: 2 Fließgewässer die Persante und die Radaune sowie 8 Siedlungsnamen, die eine der wichtigsten Fragen nach der Suche der Urheimat hervorheben, wer besiedelte dieses Gebiet im Lichte der Namenforschung. Es ist nicht einfach, vieles zu erforschen, wenn z.B. geschichtliche Kenntnisse über die Bewohner bestimmter Gebiete fehlen. Aber die Onyme und ihre richtige Deutung ersetzen nicht in einem Fall das Fehlen anderer Beweise oder Kenntnisse und können behilflich sein, siedlunggeschichtliche Schlussfolgerungen zu ziehen.

Die Besprechung der von Kilian angegebenen Onyme sollte man doch wegen dem Fehlen der schon erwähnten geschichtlichen 
Nachrichten mit dem Paradebeispiel des Namens der Persante anfangen, der weder slawisch noch germanisch ist. In Lettland ist der FlN Pèrse bekannt, in Pommern der Landschaftsnamen Pirsna und Gebiet vom Dnepr Peresna (Schmid 1981: 5).

Schmid (1987: 6) behauptet später, dass man davon ausgehen sollte, wie man die Wurzel behandelt * per- $\hat{k}_{-}$' 'graben' (in deutsch Furche) (vgl. *perk- 'graben, aufreißen' bei LIV 475f., aus dem ersichtlich wird, dass der idg. Wurzelvorschlag von Schmid nicht von den neuesten Erkenntnissen unterscheidet) oder *per-s'spritzen' (s. altisl. fors 'Wasserfall'). Der FIN Persante könnte zu den Westbalten gehören oder zu den alten Namen. Außerdem gibt es in Goryń-Gebiet Peresuta. Es ist spannend, die gebotene Erklärung von dem Namen sowie den Gedankengang von Schmid (s. 1989: 125f.) zu verfolgen und nochmals nach dem neuesten Forschungsstand zu überprüfen.

Sprachforscher und Archäologen zeigten überzeugend auf, dass sich die erste pommersche Kultur gerade auf dem erwähnten Gebiet etwa im 2. Jtsd.v. Chr. herausbildete. Somit wird die baltische Welt in ihrer materiellen und sprachlichen (Gewässernamen) Ausdrucksform früher sichtbar als die germanische.

Freilich, es ist schwer, vieles $\mathrm{zu}$ erforschen, wenn z.B. geschichtliche Kenntnisse über die Bewohner bestimmter Gebiete fehlen. Man sollte (musste) die Evolution z.B. der entstandenen Wielbark-Kultur nach Süden im Zusammenhang mit dem Lebensrhythmus der Kulturen von Nachbarn erforschen.

Der FIN Persante ist zum ersten Mal 1159 als super ripam radua... per fluuium parsandi. (CPD I 55), 1177, 1178 ...que, vehuntur per fluuium parsandi... (CPD I 104f., 110) erwähnt, später erscheinen die Formen mit -nt- 1255 Persanta, 1321 Persante, flumen Persante, d.h. Pars-: Pers-, ant-: -and-, -nd- /-nt- (s. Udolph 1990: 234-242). M.E. sind die gebuchten Varianten in den schriftlichen, geschweige von den handschriftlichen Quellen, mit größter Sorgfalt vorzustellen, in erster Linie auch mit dem Kontext, weil der „, nackte“ Name über den geringeren Aussagewert verfügt. Der genaue Hinweis auf die Quelle und ihre Zuverlässigkeit durfte nicht fehlen. Das vermisst man leider bei vielen Autoren. An dieser Stelle ist angebracht die Ausführungen von Peter Anreiter (2013: 23ff.) anzuführen, der überzeugend betont, dass besondere Aufmerksamkeit nicht nur die Wurzel, sondern auch der Derivationsmechanismus und die morphologische Struktur verdienen, sowie das markante $n t$-Suffix, das schon in der idg. Grundsprache auftritt und wie aus dem schon Gesagten klar, dass es z.B. den Illyrern sowie 
den Germanen und Balten eigen war. Udolph (ebd. 237) schlägt den Ansatz *Persantā vor. Ewa Rzetelska-Felesko und Jerzy Duma (1977: 85) gingen von der Grundform *Parsęty aus. Nach 10 Jahren berücksichtigte Rzetelska-Felesko (1987: 77f.) sehr gewissenhaft die Ergebnisse der neuesten Forschungen und erkannte den westpomoranischen Übergang -ar-> -er-, der sich in den Buchungen des Namens widerspiegelte, und hat den Namen neu gedeutet, indem sie die Erklärung aus dem Slawischen pars- poln. prosię „Ferkel“ wiederholt und sich auf die Auffassung des alteuropäischen Namens konzentriert. Die heutige poln. Namenform Parsęta vom Jahre 1951 kann kaum mit den ältesten Buchungen in Verbindung gebracht werden. In Europa gibt es Peresuta (Ukraine), Presnja (der Nebenfluss von Moskau im Gebiet von Oka), Peresna (im Gebiet von Gomel), Perosha (der rechte Nebenfluss von Oresa). In Frage kommen als Parallelbeispiele apr. Waldname 1326 Perses (Gerullis 1922: 120); apr. Siedlungsnamen 1437/38 Perselen item hat 2 fryge dienst (OF 131 161); Perselen item hat 1 frye dinst (OF 131 159); villa Persem, (OF $105145 \mathrm{v}$ ), später dt. Koppershagen, russ. Čkalovo; 1423 Persem im Kreis Pr. Eylau (OF 162 61v, 62r); 1423 Persem im Gebiet Balga (OF 162 14r) (einige gelten als Neufunde s. Blažienè 2005: 141f.; 92f.), 1411 Persing später dt. Persing, poln. Brzeżno Lyńskie in der Wojewodschaft von Allenstein; 1423 Percheln später dt. Perselen, poln. Piersele in der Wojewodschaft von Allenstein (s. Prybytek 1993: 36, 210f.). Des Weiteren s. überzeugende Beispiele in den baltischen Sprachen: vgl. die lett. Flussnamen Pè̀rse, Pèrse, Pèrrsite, Pèrrsēja, zu denen vgl. lett. pà̀rsla 'Flocke' (ME III 176f.), aslw. *nopxb (serb. pràh 'Staub'), tsch. prší 'es stiebt, sprüht'), askand. fors 'Wasserfall', aind. prș̆atá-m 'Tropfen' (LVV Paaglis - Pikgu 2003: 341f.). Vgl. den lit. FIN *Péršé, aus dem der Name von Perosha entstehen könnte, sowie die lit. FINN Peršẽ ée, Peršokšnà (Būga III 512), die weiteren lit. Gewässernamen Per̃sas, See; Parsvétas, Parsvétis, Flüsse, zu denen vgl. lit. prã-paršas, pra-peršà, pró-perša 'nicht zugefrorene Stelle auf einem sonst zugefroreren Fluss oder See; sumpfige Stelle im Wald' (Vanagas 1981: 245f.; 255). Wojciech Smoczyński sieht bei Maria Biolik (1993: 24) den Zusammenhang vom apr. Seenamen Persing, später poln. Brzeżno, mit lit. paršingas 'Ferkel-' (das Wort ist im LKŽe nicht vorhanden, am ehesten hat der durch seine sehr kühne Ideen bekannte Autor an lit. paršinga 'trächtig (Schwein)', (s. ALEW 2 736f.) gemeint) (zu pařšas 'Schwein, Ferkel', s. ALEW ebd.), vgl. die apr. ON. Parskayme, Porsekaymen später dt. Porschkeim (s. noch Biolik 1993: 183f.; Smoczyński 2000: 75; 
Blažienè 2005: 311, 154). Nach Babik (2001: 214-217) ist Persante *-ū-stämmig, $-s$ - unklar-, man könnte an einem Diminutivum denken *Parsalędyka. Die Buchung $<$ Persantua $>$ bedeutet nichts anderes als $<$ Persantica $>$. Alles würde für die praslawische Herkunft sprechen. M.W. hat sich als einer der letzten mit diesem sehr aufregenden FIN der berühmte deutsche Indogermanist und Namenforscher Albrecht Greule (2014: 401) befasst und ihn als voreinzelsprachlich-indogermanisch gedeutet. *Pers-antā gehört laut Greule $\mathrm{zu}$ einer $-s$ - Erweiterung der idg. Wurzel *per(s)'hindurchkommen, durchqueren (besonders des Wassers)' oder idg. Wurzel *pers- 'spritzen', Wenn man überlegt, wie eifrig die Erforscher fechten sollten und wie viele Speere im Kampf brechen mussten, bis die Herkunft des besprochenen Namens schließlich enthüllt sein könnte, erscheint der Weg zu der Deutung sehr lang. Wenn man nach neueren empirischen Tatsachen und Bestätigungen bei der Festellung des Namens weiterhin suchen würde, anstatt sich auf bereits vorliegende Daten zu stützen, würden es weniger Zweifel an der Herkunft des fraglichen Flussnamens geben. Wie die wissenschaftliche Erfahrung der Autorin des vorliegenden Beitrags verdeutlicht, existieren jedoch neuere Erkenntnisse in dem Bereich, wollen aber die Onomasten nicht immer besonders gern aus zeitlichen Gründen, wie die meisten angeben, in Archiven sitzen und stöbern.

Bei der Dursicht der angeführten Parallelbeispiele für die Wurzel aus den baltischen Sprachen kann man doch auf die Frage stößen, warum die baltische Herkunft des Flussnamens nicht wieder erwogen wird. Die Wurzel *pers- war doch im Altpreußischen vorhanden, es ist kaum möglich bei der Deutung des apr. Waldnamens Perses sowie der Siedlungnamen mit *pers- von dem alteuropäischen Material auszugehen. Aus verschiedensten Gründen bedürfen diese Fragen befriedigende Antwort. Man kann selbstverständlich auf viele Vergleisnamen verweisen, aber die Frage besteht, welche Rolle bei der Wiedergabe der besagten Namen die Namenbenutzer (ob das Balten, inkl. Altpreußen waren) und die Schreiber der Kanzleien, gemeint werden die Papstkanzleien und im fernen Rom ausgestellten Urkunden oder die des Deutschen Ordens, gespielt haben.

\footnotetext{
${ }^{3}$ Sogar die Namenkundler der jüngeren Generation, die in vielen Fällen der Deutung von Onymen ihre von anderen Forschern nicht wenig unterscheidende Meinung audrücken, haben unterstrichen, dass die Theorie einer alteuropäischen Hydronymie zwar nicht unumstritten ist aber bislang noch nicht schlüssig widerlegt ist. (Nübling, Fahlbusch/Heuser 2012: 223).
} 
Eine aus den Mediävisten, Historikern und Sprachkundlern bestehende Arbeitsgruppe ist eine gewisse Zeit um die neunziger Jahre des 20. Jhs. tätig gewesen und hatte sich zum Ziel gesetzt, einen interdisziplinären Projekt zu entwickeln, das sich mit der Erforschung der Onomastik des lateinisch-germanischen Europas (3.-8. Jh.) befassen musste. Das Hauptvorhaben des Projektes bestand in der Herausarbeitung von sichereren als zuvor bekannten Kriterien, die zu der Feststellung der auf die Herkunft des Namenträgers hinweisenden Merkmale verhelfen würden. Der Forschungsstand der mit der Benennung verbundenen Untersuchungen während der großen Völkerwanderung sowie im frühen Mittelalter im Ganzen, wird durch eine paradoxale Situation geschildert: die Namen des behandelten Zeitabschnitts werden einerseits kaum dokumentiert und untersucht, andererseits spielt ein individueller Name die Hauptrolle in den Diskussionen über die Herkunft des Namenträgers (Jarnut 1998: 113). Daraus entstand sich die FreiburgDuisburger Datenbank der Personennamen (DMP) (Kettemann 2008: 123-150; s. mehr in (Hrsg.) Wolfgang Haubrichs und Christa Jochum-Goldglück 2019).

Die Autorin des Beitrages betrachtet solche Projekte als Herausforderung für die Forscher des baltischen Namengutes (gemeint werden Personennamen und Toponyme), das zerstreut in ganz Europa seit der alten, sogar der ältesten Zeiten vorhanden ist und noch in Tausenden von handschriftlichen Dokumenten, Karten und anderen geschichtlichen Quellen auf den sorgfältigen Blick der Wissenschaftler wartet in der Hoffnung entdeckt und erkannt zu werden $^{4}$. Man muss im Klaren darüber sein, dass die baltische Onymie zahlenmäßig die Onymie des romanisch-germanischen Europas nicht übertreffen wird, aber ungeachtet aller Menschenverluste und verdunkelten ethnischen Verhältnisse ist dieser Versuch alle Mühe wert, um den künftigen Generationen und der Weltgeschichte zu sagen, wir haben alles gemacht, wer mehr kann, soll das machen für eine gemeinsame Zukunft der Wissenschaft, die doch im Dienste der Bedürfnisse der Menschheit stehen soll. Eine ausführliche und gewissenhafte Analyse von den Quellen würde helfen, viele Fragen zu beantworten, wenn man auf zählebige Vorurteile in der Onomastik verzichten möchte. Für sein vor kurzem

\footnotetext{
${ }^{4}$ Darius Ivoška (2016: 215-223) hat bis jetzt unbekannte oder wesentlich korrigierte Tatsachen von zwei $\mathrm{ON}$ aus dem Weichselgebiet angeführt: Pipingisse, gebucht im Jahre 1352, und Potollen vom Jahre 1352 (OF $105236 \mathrm{v}, 254 \mathrm{v}$ ).
} 
erschienenes Werk hat Jovaiša (2019) ein exaktes Motto, das in deutscher Übertragung lauten könnte: Durch die Unkenntnis zur Erkenntnis gewählt, das sogar unsere Untersuchungen zum Namengut deutlicher werden lässt.

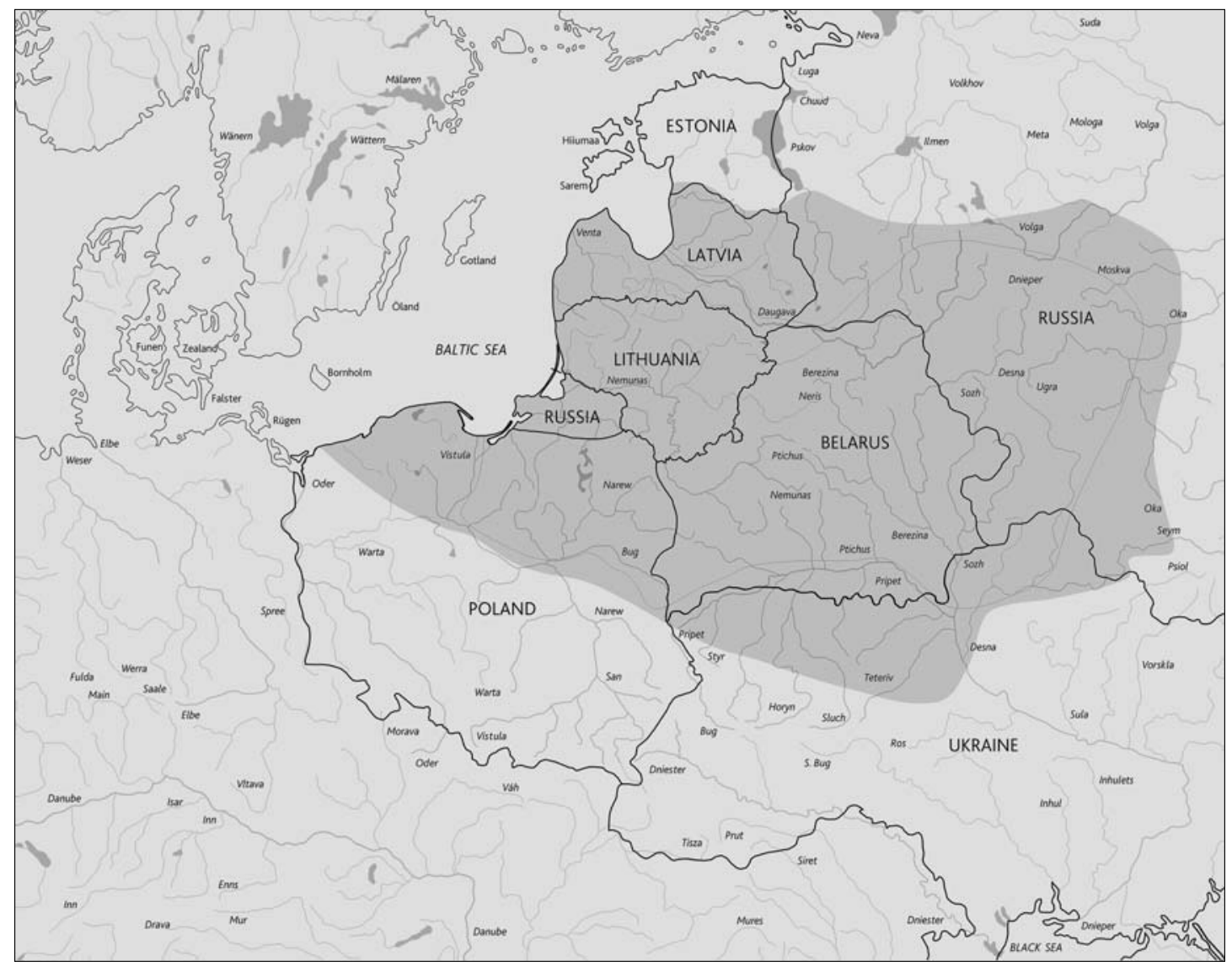

Karte Nr. 2. Baltische Gewässernamen und ihre Verbreitungsgrenzen. Autor E. Jovaiša.

\section{Literatur}

Alekna, D. 2019: Nuo Agripos iki Plinijaus: Vysla I a. romėnų geografinèje literatūroje. Lituanistica T. 65. Nr. 4 (118), 251-271.

ALEW = Altlitauisches etymologisches Wörterbuch. Unter der Leitung von Wolfgang Hock und der Mitarbeit von Elvira-Julia Bukevičiūtė und Christiane Schiller, bearbeitet von Rainer Fecht, Anna Helene Feulner, Eugen Hill und Dagmar S. Wodko. Bd. 1-3. Hamburg: Baar, 2015.

Anreiter, P. 2013: Gedanken zum Buch von Theo Venemann gen. Nierfeld, „Europa vasconica - Europa semitica“. In: Europoa Vasconica Europa Semitica? Kritische Beiträge nach dem baskischen und semitischen Substrat in Europa. Hrsg. von Jürgen Udolph. Beiträge zur lexikographie und Namenforschung. Hrsg. von Silvio Brendler Band 6. Hamburg: Baar-Verlag, 11-63. 
Babik, Z. 2001: Najstarsza warstwa nazewnicza na ziemiach polskich. W granicach wszesnośredniowieczcnej słowiańszczyzny. Kraków: Wydawnictwo Prac Naukowych UNIVERSITAS.

Beyersdorf, O. 1881: Slavische Streifen (2). In: Baltische Studien. Hrsg. von der Gesellschaft für Pommersche Geschichte und Altertumskunde, Jg. 31. Stettin: Druck und Verlag von Herrcke \& Lebeling. Anlage 25-64.

Biolik, M. 1993: Namen der stehenden Gewässer im Zuflußgebiet des Pregel und im Einzugsbereich der Zuflüsse zur Ostsee zwischen Pregel und Memel (= Hydronymia Europaea, Lief. 11). Stuttgart: Franz Steiner Verlag.

Blažek, V. 2003: Baltic Horizon in Eastern Bohemian Hydronymy? In: Vakaru baltu kalbos ir kultūros reliktai IV. Klaipėda: Tiltai 14, 1420.

Blažienè, G. 2000: Die baltischen Ortsnamen im Samland (= Hydronymia Europaea, Sonderband II). Stuttgart: Franz Steiner Verlag.

Blažienè, G. 2005: Baltische Ortsnamen in Ostpreußen (= Hydronymia Europaea, Sonderband III), Stuttgart: Franz Steiner Verlag.

Brauer, W. R. 1983: Prußische Siedlungen westlich der Weichsel. Versuch einer etymologischen Deutung heimatlicher Flurnamen. Siegen: Im Selbstverlag der J. G. Herder-Bibliothek Siegerland e. v.

Brauer, W. R. 1988: Baltisch-preussische Siedlungen westlich der Weichsel. Münster: Nicolaus-Copernicus-Verlag.

Būga, K. 1923/1961: Upių vardų studijos ir aisčių bei slavėnų senovė. Rinktiniai Raštai T. 3. Vilnius: Valstybinè politinès ir mokslinès literatūros leidykla, 493-550.

Casemir, K., Udolph, J. 2006: Die Bedeutung des Baltischen für die niedersächsische Ortsnamenforschung. In: Baltu onomastikos tyrimai. Vilnius: Lietuviu kalbos institutas, 114-144.

CPD - Codex Pomeraniae Diplomaticus oder Sammlung der die Geschichte Pommerns und Rügens betreffenden Urkunden. Nach den Originalen, Transsumten und alten Copieen mit Anmerkungen, Schriftproben und Siegelzeichnungen. Hrsg. von Karl Friedrich Wilhelm Hasselbach, D. Johann Gottfried Ludwig Kosegarten und Friedrich Baron von Medem. Bd. 1. Greifswald: Universitätsbuchhandlung C. A. Koch, 1843.

Curta, F. 2001: The Making of the Slavs: History and Archeology of the Lower Danube Region, c. 500-700 (Cambridge Studies in Medieval Life and Thougth. Fourth Series). Publisher: Cambridge University Press.

Dambe, V. 1988: Daži hipotēski baltismi vietu nosaukumos rietumos no Vislas. In: Baltistica XXIV (1), 76-79.

Dini, P. U. 2019: Baltu kalbu lyginamoji istorija. Vilnius: Mokslo ir enciklopedijų leidybos centras.

DONB = Matusiak-Kempa, I. 2012: Danzig // Gdańsk. In: Deutsches Ortsnamenbuch. Hrsg. von Manfred Niemeyer. Berlin; Boston: Walter de Gruyter GmbH \& C. KG, 119.

Gimbutienè, M. 1985: Baltai priešistoriniais laikais. Etnogenezè, materialinè kultūra ir mitologija. Vilnius: Mokslas. 
Gerullis, G. 1922: Die altpreußischen Ortsnamen. Berlin; Leipzig: Walter de Gruyter \& Co.

Gillett, A. 2002: Introduction: Ethnicity, History, and Methodology. In: Gillett Andrew (ed.). On Barbarian Identity: Critical Approaches to Ethnicity in the Early Middle Ages. Studies in the Early Middle Ages, Vol. 4. Turnhout: Brepols Publishers n.v., 1-18.

Gillett, A. 2002: Was Ethnicity Politicized in the Earliest Medieval Kingdoms? In: Gillett Andrew (ed.). On Barbarian Identity: Critical Approaches to Ethnicity in the Early Middle Ages. Studies in the Early Middle Ages, Vol. 4. Turnhout: Brepols Publishers n.v., 85-121.

Goffart, W. 2002: Does the Distant Past Impinge on the Invasion Age Germans? In: Gillett Andrew (ed.). On Barbarian Identity: Critical Approaches to Ethnicity in the Early Middle Ages. Studies in the Early Middle Ages. Vol. 4. Turnhout: Brepols Publishers n.v., 21-37.

Górnowicz, H. 1980: Toponimia Powiśla Gdanskiego. Wrocław: Zakład Narodowy im. Ossolińskich.

Górnowicz, H. 1985: Gewässernamen im Flußgebiet der unteren Weichsel (Nazwy wodne dorzeca dolnej Wisły) (= Hydronymia Europaea, Lief. I). Wiesbaden GMBH, Stuttgart: Franz Steiner Verlag.

Graus, F. 1980: Die Nationalbildung Westslawen im Mittelalter (Nationes: Historische und philologische Untersuchung zur Entstehung der europäischen Nationen im Mittelalter, number 3). Sigmaringen. Stuttgart: Jan Thorbecke Verlag.

Greule, A. 2014: Deutsches Gewässernamenbuch. Etymologie der Gewässernamen und der zugehörigen Gebiets-, Siedlungs- und Flurnamen. Berlin; Boston: Walter de Gruyter GmbH \& co. KG.

Haubrichs, W., Jochum-Goldglück, Ch. (Hrsg.) 2019: Kulturelle Integration und Personennamen im Mittelalter. Berlin; Boston: Walter de Gruyter. (= Ergänzungsbänbe zum Realllexikon der Germanischen Altertumskunde 108).

Hengst, K. 1985: Sprachkontakt und Entlehnungsprozeß. Ergebnisse der toponomastischen Analyse im deutsch-slawischen Berührungsgebiet. In: Zeitschrif für Slawistik 30, 6, 809-822.

Hinze, F. 1989: Rez. W. Brauer. Preußische Siedlungen westlich der Weichsel. Versuch einer etymologischen Deutung heimatlicher Flurnamen. Schriften der J. G. Herder-Bibliothek Siegerland e.V. Bd. 11. 1983. Selbtsverlag der J. G. Herder-Bibliothek Siegerland e.V. Siegen 1983. 91 (+3) S. In: Zeszyty naukowe wydziatu Humanistycznego uniwersytetu Gdańskiego. Prace Językoznawcze Nr. $15,129-140$.

Holsten, R. 1932: Illyrische Fluß- und Ortsnamen in Pommern. In: Monatsblätter der Gesellschaft für pommersche Geschichte und Altertumskunde Jg. 46., Nr. 1, 1-5.

Isajiw, W.W. 1993: Definition and Dimensions of Ethnicity. In: A Theoretical Framework. Challenges of Measuring an Ethnic World: Science, politics and reality: Proceedings of the Joint CanadaUnited States Conference on the Measurement of Ethnicity April 13, 1992, Statistics Canada and U.S. Bureau of the Census, eds. Washington, D.C.: U.S. Government Printing Office, 407-427. (Paper presented at "Joint Canada-United States Conference on the 
Measurement of Ethnicity", Ottawa, Ontario, Canada, April 2, 1992).

Ivoška, D. 2016: Bemerkungen zur Problematik der baltischen Ortsnamen westlich der Weichsel. In: Acta Linguistica Lithuanica LXXIV, 208-225.

Jarnut, J. 1998: Nomen et Gens: Political and Linguistic Aspects of Personal Names between the Third and the Eighth Century - Presenting an Interdisciplinary Project from a Historical Perspective. In: Pohl, Walter \& Reimitz, Helmut (eds.) Strategies of Distinction: The Construction of Ethnic Communities, 300-800. Leiden; Boston; Köln: BRILL, 113-116.

Jovaiša, E. 2012 「Nachdruck 20131: Aisčiai. Kilmé. Vilnius: Lietuvos edukologiios universiteto leidykla.

Jovaiša, E. 2014: Aisčiai. Raida. Vilnius: Lietuvos edukologijos universiteto leidvkla.

Jovaiša, E. 2019: The Aestii. The Western Balts. Kaunas: Vytautas Magnus University.

Jovaiša, E. 2019: Kapai ir žmonès. Aisčiai, romènai, gotai ir kitos istorijos. Vilnius: UAB ,Unseen Pictures“.

Kettemann, W. 2008: Name, Person, Gruppe. Potential und Entwicklungsmöglichkeiten der Freiburg-Duisburger Datenbank mittelalterlichen Personennamen (DMP) für künftige Forschungvorhaben. In: Consilium medii aevi 11, 123-150.

Kilian, L. 1939: Baltische Ortsnamen westlich der Weichsel? In: Alt-Preußen 4, 67-68.

Kilian, L. 1960: Zum Aussagewert von Fund- und Kulturprovinzen. In: Światowit 23, 41-85.

Krahe, H. 1943: Baltische Ortsnamen westlich der Weichsel. In: AltPreußen 8, 11-12.

Kulikowski, M. 2002: Nation versus Army: A Necessary Contrast? In: Gillett Andrew (ed.). On Barbarian Identity: Critical Approaches to Ethnicity in the Early Middle Ages. Studies in the Early Middle Ages, Vol. 4. Turnhout: Brepols Publishers n.v., 69-84.

Kusmenko, J. K. 2011: Rannije germancy $i$ ich sosedi. Lingvistika, archeologija, genetika. Sankt-Peterburg: „,Nestor-Istorija“.

Lorentz, F. 1933: Preußen in Pomerellen. In: Mitteilungen des Westpreußischen Geschichtsvereins Jg. 32, Heft 3, 49-59.

Lorentz, F. 1966: Preußische Ortsnamen und Appellative in Namen im Raum links unter der Weichsel mit Anmerkungen von F. Hinze. In: Zeitschrift für Slawistik XI, 243-250.

LIV - Lexikon der indogermanischen Verben. Die Wurzeln und ihre Primärsstammbildungen. Unter Leitung von Helmut Rix. Zweite, erweiterte und verbesserte Auflage bearbeitet von Martin Kümmel und Helmut Rix. Wiesbaden: Dr. Ludwig Reichert Verlag, 2001.

LKŽe - Lietuvių kalbos žodynas 1-20, 1941-2002, elektroninis variantas, redaktorių kolegija: Gertrūda Naktinienè (vyr. red.), Jonas Paulauskas, Ritutè Petrokienè, Vytautas Vitkauskas, Jolanta Zabarskaitè. Vilnius: Lietuvių kalbos institutas, 2005 (atnaujinta versija, 2008). Zutritt: http://www.lkz.lt/>www.lkz.lt. 
LVV — Mülenbachs, K. 1926-1932: Latviešu valodas vārdnīca. Rediǵējis, papildinājis, turpinājis J. Endzelīns, Bd. 1-4, Rīga: Izglitības ministerija, Kultūras fonds.

Murray, A.C. 2002: Reinhard Wenskus on "Ethnogenesis", Ethnicity, and the Origin of the Franks. In: Gillett Andrew (ed.). On Barbarian Identity: Critical Approaches to Ethnicity in the Early Middle Ages. Studies in the Early Middle Ages, Vol. 4. Turnhout: Brepols Publishers n.v., 39-68.

NMP III - Nazwy miejscowe polski. T. III E-I (pod redakcja Kazimierza Rymuta). Kraków: Wydawnictwo Naukowe DWN, 1999.

NMP IV - Nazwy miejscowe polski. T. IV J-Km (pod redakcja Kazimierza Rymuta). Kraków: Wydawnictwo Naukowe DWN, 2001.

NMP VII - Nazwy miejscowe polski. T. VII Mą-N (pod redakcją Kazimierza Rymuta). Kraków: Wydawnictwo Pandit, 2007.

Nübling, D., Fahlbusch, F., Heuser, R. Namen. Eine Einführung in die Onomastik. Tübingen: Narr Francke Attempto Verlag GmbH + Co.

Pohl, W. 1998: Telling the Difference: Signs of Ethnic Identity. In: Pohl, Walter \& Reimitz, Helmut (eds.). Strategies of Distinction: The Construction of Ethnic Communities 300-800. (The Transformation of the Roman world. Vol. 2). Leiden; Boston; Köln: BRILL, 17-69.

Pohl, W. 2002: Ethnicity, Theory, and Tradition: A Response. In: Gillett Andrew (ed.). On Barbarian Identity: Critical Approaches to Ethnicity in the Early Middle Ages. Studies in the Early Middle Ages. Vol. 4. Turnhout: Brepols Publishers n.v., 220-239.

Przybytek, R. 1993: Ortsnamen baltischer Herkunft im südlichen Teil Ostpreußens. Stuttgart: Franz Steiner Verlag. (= Hydronymia Europaea, Sonderband).

Rzetelska-Felesko, E., Duma, J. 1977: Nazwy rzeczne Pomorza miedz.v dolna Wista a dolna Odra. Wrocław: Zakład Narodowy im. Ossolińskich.

Rzetelska-Feleszko, E. 1987: Die Zuflüsse zur Ostsee von der Weichselmundung bis zur Persante (Dopływy Bałtyku od ujścia Wisty do Parsęty). Wiesbaden: GMBH, Stuttgart: Franz Steiner Verlag (= Hydronymia Europaea, Lief. 2).

Schmid, W. P. 1981: Die alteuropäische Hydronymie. Stand und Aufgaben ihrer Erforschung. Beiträge zur Namenforschung N. F. 16, 1-12.

Schmid, W. P. 1987: Beiträge zur Bestimmung der baltischen Westgrenze. In: Baltistica 23, 4-12.

Schmid, W. P. 1989: Spuren baltischer Sprache in Pommern. In: Rymut, K. (red.). Hydronimia stowiańska. Materiały z IX konferencsji Komisji Onomastyki Słowiańskiego przy Międzynarodovym Komitecie Sławistów. Mogilany, 16-18 IX 1986 r. Wrocław; Warszawa; Kraków; Gdańsk; Łódż, 119-128.

Smoczyński, W. 2000: Untersuchungen zum deutschen Lehngut im Altpreussischen. Kraków: Wydawnictwo Uniwersitetu Jagiellońskiego.

Szcześniak, K. 1993: Unbekannte baltische Bezeichnungen aus dem Goldaper Kreis im Thorner Nachlass von Martin Gerss. In: Lietuviu kalbotyros klausimai XXXII, Baltų onomastikos tyrinèjimai, 166-171. 
Toporov, V. N. 1966: K voprosu o toponimičeskich sootvetstvijach na baltijskich territorijach i k zapadu ot Vistly. In: Baltistika I (2), S. 103-111.

Toporov, V. N. 1983: Novyje raboty o sledach prebyvanija prussov $\mathrm{k}$ zapadu ot Visly. In: Balto-slavjanskie issledovanija 1982, 263-273.

Toporov, V. N. 1984-1990: Prusskij jazyk. Slovar'. T. 4-5, Moskva: Nauka.

Trautmann, R. 1925: Die altpreußischen Personennamen. Göttingen: Vandenhoeck \& Ruprecht.

Udolph, J. 1988: [Rez.]: Wilhelm Brauer. Prußische Siedlungen westlich der Weichsel. Versuch einer etymologischen Deutung heimatlicher Flurnamen. Schriften der J. G. Herder-Bibliothek Siegerland e.V. Band 11. 1983. Selbtsverlag der J. G. Herder-Bibliothek Siegerland e.V. Siegen. 94 S. $8^{0}$. In: Beiträge zur Namenforschung. Neue Folge 23, 291-300.

Udolph, J. 1990: Die Stellung der Gewässernamen Polens innerhalb der alteuropäischen Hydronymie. Heidelberg: Carl Winter Universitätsverlag.

Udolph, J. 2014: Ethnogenese und Urheimat der Slaven. In: Die slavischen Sprachen, The Slavic Languagues. Ein internationales Handbuch zu ihrer Struktur, ihrer Geschichte und ihrer Erforschung / An International Handbook of their Structure, their History und their Investigation. Hrsg. von Karl Gutschmidt, Sebastian Kempgen, Tilman Berger, Peter Kosta, Bd. 2. Berlin; Munich; Boston: Walter de Gruyter GmbH, 1131-1144.

Vanagas, A. 1981: Lietuviu hidronimu etimologinis žodynas. Vilnius: Mokslas.

Vasmer, M. 1930: [Rez.] Über J. Czekanowski, Kritisches und Antikritisches zu M. Vasmers Besprechungen usw. In: Zeitschrift für slavische Philologie 7, 504-507.

Wenskus, R. 1961 (1977): Stammesbildung und Verfassung: das Werden der frühmittelalterlichen gentes. Köln: Böhlau Verlag.

Wenzel, W. 2015: Namenkundliche Beiträge zur Siedlungsgeschichte des Klosters Dobrilugk. In: Slawen in Deutschland. Ihre Namen als Zeugen der Geschichte. Hrsg. von Andrea Brendler und Silvio Brendler. Hamburg: Baar-Verlag, 30-41.

Wolfram, H. 2001 (2010): Die Goten und ihre Geschichte. 3. durchgesehe Aufl. München: C. H. Beck Verlag. (In der Beck'schen Reihe Wissen).

Wolfram, H. 2008: Terminologisches. In: Nomen et Fraternitas. Festschrift für Dieter Geuenich zum 65. Geburtstag. Hrsg. von Uwe Ludwig und Thomas Schilp. Berlin; New York: Walter de Gruyter, 787802.

Wolfram, H. 2011: Wie viele Völker sind in einem Volk? In: Studies in honour of Professor Vassil Giuzelev [= Bulgaria Mediaevalis 2], Sofia: Bulgarian Historical Heritage Foundation, 361-372.

Ziemann, D., Trencsényi, B. 2011: Ethnogenesis, Nation, Nation-Bulding. Zutritt: http://archive.ceu.edu/node/24561 